\title{
Towards a holistic approach of patients with Asthma and COPD: implementing a diagnostic care pathway
}

Jan A. Witte ( $\square$ adjanwitte@gmail.com )

Franciscus Gasthuis en Vlietland https://orcid.org/0000-0002-9037-1002

Erwin Birnie

Franciscus Gasthuis en Vlietland

\section{Gert-Jan Braunstahl}

Franciscus Gasthuis en Vlietland

\section{Edmée van den Akker}

Franciscus Gasthuis en Vlietland

Walter J.M. van Litsenburg

Catharina Ziekenhuis

\section{Niels H. Chavannes}

Leiden Universitair Medisch Centrum: Leids Universitair Medisch Centrum

Maureen P.M.H. Rutten - Van Mölken

Erasmus Universiteit Rotterdam Instituut Beleid en Management Gezondheidszorg

Johannes C.C.M. in 't Veen

Franciscus Gasthuis en Vlietland

Research article

Keywords: Holistic Health, Practice Guideline, Delphi Technique, Asthma, COPD, Health Plan Implementation

Posted Date: December 10th, 2020

DOI: https://doi.org/10.21203/rs.3.rs-124416/v1

License: (c) (1) This work is licensed under a Creative Commons Attribution 4.0 International License. Read Full License 


\section{Abstract \\ Objective}

To evaluate implementation of recent guidelines in day-to-day clinical practice in patients with Obstructive Lung Disease (OLD).

\section{Methods}

Guidelines recommend attention for four important domains (Physiological impairments, Symptoms, Functional Limitations and Quality of life) as they give important clues for evaluation of treatment outcomes and for developing new treatments. A new diagnostic care pathway was developed to attain a holistic characterization of patients with OLD. Here, we assessed the implementation of this pathway at our outpatient clinic in secondary care in a controlled, cohort study, by comparing the diagnostic items in patients with OLD before (2013) and after (2015) implementation. Results were compared with reference data (2016) from two control hospitals.

\section{Results}

The diagnostic care pathway provided a significant increase in overall attention for aforementioned domains from $52 \%$ (median; IQR 44\%-59\%) to $81 \%$ (median; IQR 74\%-85\%). Especially the domains Quality of Life and Functional Limitation benefitted from the diagnostic care pathway by improving from $0-100 \%$ and 0 to $67 \%$ (median; IQR $100 \%-100 \%$ and $67 \%-83 \%$ ) respectively. Approximately $65 \%$ of the patients failed to complete the 2-day assessment; they received usual care. Nonetheless, attention for the four-domain approach also improved in these patients receiving usual care (median, IQR; $52 \%$ (44-59) vs 69\% (59-74). Additionally, $14 \%$ of new referrals for diagnosis and treatment of OLD did not have asthma or COPD after thorough assessment in secondary care.

\section{Conclusion}

The implementation of current guidelines in daily practice can achieve a culture change among healthcare professionals. However, implementing a care pathway in a real-live population proved difficult. Therefore, we should strive to successfully implement existing guidelines rather than primarily creating new guidelines, as implementation is one of the most challenging aspects of new pathways and protocols.

\section{Contributions To The Literature}

- Study results emphasize the importance on implementing current guidelines and protocols on OLD, rather than focussing on updating current guidelines.

- Implementation of current guidelines on diagnostic strategies improves healthcare in participating as well as non-participating healthcare professionals.

- Optimal referral strategies between primary and secondary care need further study to ensure tailor made management in novel diagnostic pathways.

\section{Introduction}

Asthma and COPD are highly prevalent, chronic obstructive lung disorders often touching several and variable facets of the lives of affected people, which are poorly captured and explained based on physiological measurements only $(1,2)$. Recent GOLD guidelines underlines that 'OLD impacts patients beyond dyspnoea(2).' Therefore, an adequate analysis of health status in patients with chronic lung conditions should not only comprise an evaluation of the physiological impairment(s), including potential systemic effects and comorbidities, but should also incorporate a thorough assessment of the symptoms, patient's functional limitation and his/hers quality of life (3-9). GOLD guidelines emphasize the importance of implementing new strategies with a holistic approach. However, the available literature on implementation of this approach is scarce. Our study aims to close this gap, by implementing a new diagnostic pathway in daily practice in the general population of patients with OLD.

Recently we developed and implemented an innovative diagnostic care pathway for patients with obstructive lung disease (10). The aim of this diagnostic pathway is to perform an integral, holistic assessment of the health status of patients referred to secondary care. We 
developed a practice-based diagnostic pathway, in which we implemented diagnostic items and instruments as described previously (11). This diagnostic pathway consists of an evidence-based set of measurements prioritized by a Delphi procedure. This set includes traditional items, such as prior history, physical examination, spirometry, blood gasses and X-ray but adds three innovative diagnostics: the metronome-paced hyperventilation test to measure dynamic hyperinflation, an activity monitor to objectively evaluate physical activity in daily life, and the Nijmegen Clinical Screening Instrument as a comprehensive assessment tool to acquire detailed insight into symptoms, functional limitations, and quality of life (10). With these diagnostic items we aim to identify treatable traits which are essential for providing a tailor made treatment. $(3,12)$

In this study we review the implementation of this strategy. The aim is twofold. Our first aim was to determine the feasibility of the pathway as the percentage of patients that succesfully completed the program, as compared to the whole eligible patient population. The second goal was to evaluate the number of assessed diagnostic items per patient as compared to the pre-implementation period. For both goals, $50 \%$ was defined as a successful implementation of the diagnostic pathway.

\section{Methods}

\section{- Study design}

in a controlled, but not randomized, cohort study we compared the comprehensiveness of the diagnostic pathway in patients with asthma or COPD before (2013) and after (2015) the implementation period of the diagnostic pathway at the pulmonology outpatient clinic, Franciscus Gasthuis, Rotterdam, the Netherlands.

During the implementation of the pathway, the most recent national guidelines (7-9) were disseminated nationwide. Therefore, quality of healthcare is expected to increase over time in all hospitals regardless of the implementation of the new pathway. To adjust for this phenomenon, we compared our outcomes with the metadata of two control hospitals (2016) that did not implement the pathway but adopted general (inter)national services concerning integrated care of Asthma $(1,13)$ and $\operatorname{COPD}(2,8)$. These two control hospitals, in the north and south-west of the Netherlands, are comparable to the intervention hospital with respect to their focus on obstructive airways disease and participation in similar (nationwide) projects to improve care for COPD patients.

\section{- Novel items of the Diagnostic pathway}

The diagnostic care pathway was based on the Dutch standards of care for patients with Asthma and COPD as described by the Dutch Lung Alliance $(\operatorname{LAN})(7,8)$. These guidelines are in agreement with international guidelines, especially the assessment of patients with asthma and COPD. These care standards are based on a four-domain model : physiological impairment, symptoms, functional impairment and quality of life (see Figure 1). A two-day stepped diagnostic pathway was made, creating a structured holistic approach, using the domains and diagnostic items in Figure 1 as standardized measurements (10). Three major innovations were added to the existing domains and diagnostic items. Firstly, the metronome-paced hyperventilation test to measure dynamic hyperinflation. Secondly, a physical activity monitor to objectively evaluate physical activity in daily life during seven days. Thirdly, the Nijmegen Clinical Screening Instrument as standardised survey (14). This in-depth view helps to identify individual treatable traits and motivate patients to change their behaviour.

\section{- Patients and records}

Earlier secondary care studies of obstructive lung diseases had highly specific selection criteria, which made it difficult to extrapolate the results to a large 'real life' population (15). We therefore choose to include all patients with suspected asthma, COPD or Asthma-COPD Overlap (ACO). The electronic medical records of all new patients referred by the general practitioner with probable asthma, COPD or ACO were identified six months before (January-June 2013) and one and a half year after (January-June 2015) the implementation of the diagnostic care pathway. Patients with incorrect referral (defined as not referred for diagnoses and treatment of OLD), patients that had been diagnosed previously or patients who visited the pulmonologist for another reason, such as pre-operative assessment or monitoring after hospital admission were excluded.

For each period, 100 medical records of patients referred to the outpatient clinic were selected by an independent researcher using a random number generator.

In the two control hospitals, medical records of patients diagnosed with asthma, COPD or ACO in the period January-June 2016 were analysed. In those control hospitals, medical records were grouped per pulmonologist and every fifth medical record was analysed. The 
numbers of records analysed are shown in Figure 2.

In the intervention hospital data was collected by a research physician assistant (research-PA). In the control hospitals the data was collected by a research nurse specialist (research-NS). To minimalize differences between data collectors, a data protocol was made, describing the definitions of all variables, called Integral Practice Analyses Trajectory in secondary care (IPAT-2) after which the inter-rater agreement was assessed. In both intervention and control hospitals 50 randomly selected cases were assessed by either research-PA or research-NS and an independent researcher.

Figure 2.

\section{- Characteristics of data registry}

Table 2 shows that in the period before implementation of the diagnostic pathway (e.g. 2013), 476 patients had been referred to the pulmonologist suspected for asthma and COPD. After the implementation (2015) the number of referred patients was 413 . After applying the in- and exclusion criteria 101 medical records from 2013, divided over five different pulmonologists, were randomly chosen to be included. In 201593 patients met the inclusion criteria; they were all included.

In the control hospitals every fifth patient with suspected OLD was selected by the administrative department, resulting in the inclusion of 50 and 57 medical records from the two control hospitals.

\section{- Study endpoints}

To study the feasibility of the pathway we determined the percentage of patients succesfully completing the program.

We assessed the level of implementation in two steps: we calculated the number of items assessed per patient (as an indicator of the level of implementation) and the number of patients assessed per item (as an indicator of which items were poorly implemented) This provides two indicators for the level of implementation.

\section{- Statistics}

\section{- Analysis of the test-retest study}

Data of research-PA and research-NS were compared using the data of the independent researcher to correct for differences between the hospitals. Cohen's Kappa was used to measure inter-observer agreement.

\section{- Statistical analysis}

The number of diagnostic items assessed per patient was calculated and compared between the diagnostic care pathway group (2015) and baseline (2013) using the Mann-Whitney U Test and the Fisher's Exact Test.

The percentage of patients per individual diagnostic item (Figure 1) was assessed descriptively and no statistical testing was performed with this data.

To adjust for national trends concerning quality of healthcare, we used data of two hospitals as controls. These hospitals generously provided meta-data and means. Because we had no raw data, medians or other summarizing data could not be calculated. The group included in the care pathway (2015) was compared to the control hospitals (2016) using the one-sample Student's t-test.

\section{- Ethical approval}

The Medical Research Ethics Committee TWOR declared that this study has no conflict with the Medical Research Involving Human Subjects Act (in Dutch called 'WMO') and gave a waiver that neither medical ethical approval nor written informed consent from participants was needed for this registry-based study.

\section{Results}

\section{- Analysis of data registration}


The inter-rater reliability was substantial with a Cohen's Kappa 0.7. A test-retest analysis was done by both PA and NS, with a Cohens Kappa of subsequently 0.97 and 1.00 (a Cohen's kappa 0.81 - 1.00 indicates almost perfect agreement)(16).

\section{- Characteristics of diagnostisch assessment}

After implementing the diagnostic care pathway, 93 patients suspected for asthma, COPD or ACO were invited to be assessed using the new diagnostic pathway. A total of 33 patients (35\%) participated in the study design and were diagnosed using the novel diagnostic pathway. The remaining 60 patients (65\%) dropped out and and were instead assessed in the traditional way, which was based on the individual opinion of the attending pulmonologist. Therefore, a distinction was made between patients who were assessed according to the structured two-day stepped pathway $(n=33)$ and those who were not. Approximately $14 \%$ of new referrals for diagnosis and treatment of OLD did not have asthma or COPD after thorough assessment in secondary care.

\section{- Assessed diagnostic items per patient}

The median number of measured diagnostic items per patient was significantly increased in the group included in the care pathway as compared to baseline (see table 1). The increase was significant for all domains, especially in the domains 'quality of life' and 'functional limitations'. The registration in these two domains was also increased in the group receiving standard care in the intervention hospital. The group included in the care pathway scored significantly more items/assessments on all domains compared to the group receiving usual care in the control hospitals (see table 2).

\section{- Assessed patients per diagnostic item}

Figure 3 shows the percentage of patients in which a diagnostic item was assessed and reported. Improvements were seen, both in the group included in the pathway and in the patients receiving usual care. The increase was higher though in the patients included in the pathway: Items registered more frequently were exacerbations (from $6 \%$ to $76 \%$ ), dynamic hyperinflation (from $2 \%$ to $94 \%$ ), physical activity objective (from $2 \%$ to 100\%) and physical activity subjective (from 13\% to 100\%) and quality of life in asthma patients (from $17 \%$ to $97 \%$ ) and quality of life in COPD patients (from $13 \%$ to $100 \%$ ). Although the diagnostic items 'comorbidity' and 'pulmonary history' were registered more frequently in patients assessed in the newly implemented care pathway and traditionally assessed patients scored even higher percentages. In contrast, both in the group included in the pathway and in the group receiving usual care, the diagnostic items 'lung volume' (from $83 \%$ to 55\%) and 'diffusion capacity' (from $43 \%$ to $18 \%$ ) were registered less frequently. This is the result of the 2016 Delphi Panel Study which demonstrated that lung volume and diffusion capacity weren't conceived as treatable traits, after which these items were no longer part of the diagnostic care pathway.(11)

\section{Discussion}

Obstructive lung disease (OLD) is a highly prevalent and chronic disorder touching numerous aspects of the lives of affected people. Literature and guidelines advocate the importance of identifying individual treatable traits to improve wellbeing of these patients (1-3, 5, $6,10,11)$. However, the available literature on implementation of this approach is scarce. Our study aims to close this gap, and emphasizes the difficulty of implementing a new diagnostic pathway in daily practice in the general population of patients with OLD. Notwithstanding that only one third of the patients completed the assessment, there was an increase in measured and documented domains in both patients who completed the assessment as well as the patients who followed usual care.

To our knowledge, the implementation of a diagnostic process (e.g. operationalisation of guidelines) of patients with asthma and/or COPD in secondary care has never been analysed in such a detailed way. Only recently, MacDonald and co-workers studied the potential added value of a treatable trait approach in severe asthma (17). This study included both a crossover and randomized controlled trial (RCT). However, the RCT included a highly selected subset of patients with OLD, which makes the study poorly comparable to a real world population. For this reason, we aimed to review the level of implementation in a real world population with patients of OLD.

Many studies and guidelines describe the importance of a holistic approach in the diagnostic process and even define four important domains, but lack a description of the content (1-5). Previously, we determined the diagnostic items that should be part of a holistic assessment in secondary care; here we developed the registry to evaluate whether these diagnostic items were measured in a systematic way, and analysed the change in the diagnostic process after implementation of a diagnostic care pathway. The results confirm that this approach increases attention for the four important domains, especially for the domains of functional limitation and quality of life. 
It proved to be challenging to create a pathway with a more than $50 \%$ successful completion rate. About one third of the patients successfully completed the diagnostic process. Although no data are available about the reasons of not accepting the invitation to this study by patients, one explanation might be that patient with more progressed OLD have difficulties to attend the two day assessment. Another explanation might be the lack of motivation in patients to undergo this in depth assessment. In the future, the pathway will be adjusted to address the motivation, opportunity and capacity of patients with OLD according to the Poiesz triad (18). To that end, we also will focus on the motivational status and willingness to change by incorporating the Patient Activation Measure (PAM) questionnaire. A recent study on Parkinson's disease noticed this problem as well and introduced a digital format to measure patient 'self-reported outcomes'. In that pathway, less than $14 \%$ of the participants completed the program (19). The factors contributing to their disappointing numbers were related to both medical staff, logistics and patients. For example, insufficient time was allocated for the study procedure was allocated for visits of the outpatients clinic, which resulted in poor adherence to the study protocol was poor. In our study we minimalised the factors caused by medical staff by scheduling educational meetings, but found it challenging to minimalize patient related- and logistic-factors. Although we did not register the specific reasons, non-participation of patients was most often due to the intensity of assessment or rigidity of the appointments.

Approximately $14 \%$ of referred patients could not be diagnosed with either asthma and/or COPD. This demonstrates the added value of pulmonary diagnosis in secondary, as this prevents chronic mistreatment of symptoms in these patients. Earlier studies revealed up to $30 \%$ of misdiagnosis in primary care for asthma and/or COPD (20) despite the availability of spirometry (21). This leads to incorrect referral questions to secondary care and subsequently results in patients mistakenly being included in this pathway. More attention should be paid to optimal referral conditions, as we investigated in an earlier report (11).

The registration of nearly all important domains and their components increased significantly after implementation of the pathway. Interestingly, the group included in the care pathway (2015) received a significantly superior assessment when compared to both the baseline group in the intervention hospital (2013) and the patients receiving usual care in the control hospitals (2015). These data are robust, as data of two other additional control hospitals confirmed that the improved results are not due to national trends regarding healthcare. Data of these control hospitals were provided by hospitals who used the Ipat-2 for internal use. Regrettably, legislation prevents the use of detailed data at the patient level. All hospitals provided healthcare according to recent guidelines and literature. The superior results therefore were most likely caused by implementing the new diagnostic approach. It also supports the added value of the newly implemented approach even for usual care.

The outcomes of the patients in the Care Pathway and the patients receiving usual care were comparable. Apparently, when compared to the control hospitals, healthcare in the intervention hospital improved, even when patients received standard care. This could be explained by both a cross-over effect between pulmonologists and by a change in mindset of the whole team involved. This emphasizes the importance of education and change in attitude of the medical team, to successfully implement new tests or pathways.

\section{Conclusion}

In this study we did not intend to create new guidelines, but we rather strove to implement current standards in day-to-day practice, using a new holistic diagnostic approach. We demonstrated that, even with just a part of the intended patients completing the diagnostic pathway, the assessment became much more holistic, both in patients completing the diagnostic assessment as well as patients not completing the diagnostic pathway. This shows that culture change among pulmonologists can be achieved. On the other hand, we illustrated the difficulty of creating a new diagnostic pathway with more than $50 \%$ participation in the population of patients with OLD. This demonstrates that we should not only focus on making new guidelines, but we should also aspire to successfully implement existing guidelines, as this is one of the most challenging aspects of new pathways and protocols.

\section{Declarations}

\section{- Conflict of interest statement for all authors}

The authors declare no conflict of interest.

\section{- Ethics approval and consent to participate}

The Medical Research Ethics Committee TWOR declared that this study has no conflict with the Medical Research Involving Human Subjects Act (in Dutch called 'WMO') and gave a waiver that neither medical ethical approval nor written informed consent from 
participants was needed for this registry-based study.

\section{- Consent for publication}

N/A

\section{- Availability of data and material}

The data that support the findings of this study are available from Franciscus Gasthuis en Vlietland but restrictions apply to the availability of these data, which were used under license for the current study, and so are not publicly available. Data are however available from the authors upon reasonable request and with permission of all authors.

\section{- Competing interests}

N/A

\section{- Funding}

Picasso voor COPD, Astra Zeneca, Stichting Coolsingel, Marga Roubos heritage fund

- Authors' contributions

JAW writing of manuscript and submission

EB Statistics

GJB Review of manuscript, study design

EA Creating crude version of manuscript

WJML Creating dataset in control hospital

NHCh Review of manuscript, study design

MPMHR Review of manuscript, study design, interpretation of data

JCCMV Review of manuscript, writing of manuscript, funding

\section{- Acknowledgements}

\section{References}

1. Global Initiative for Asthma. Global Strategy for Asthma Management and Prevention, 2020 Report. [cited 2020 jun 22]. Available from: https://ginasthma.org/gina-reports/.

2. Global Initiative for Chronic Ostructive Lung Disease. Global Strategy for prevention, diagnosis and management of chronic obstructive pulmonary disease, 2020 Report. [22-06-2020]. Available from: https://goldcopd.org/wpcontent/uploads/2019/12/GOLD-2020-FINAL-ver1.2-03Dec19_WMV.pdf.

3. Agusti A, MacNee W. The COPD control panel: towards personalised medicine in COPD. Thorax. 2013;68(7):687-90. doi:10.1136/thoraxjnl-2012-202772

4. Nici L, ZuWallack R. An official American Thoracic Society workshop report: The integrated care of the COPD patient. Proceedings of the American Thoracic Society. 2012;9(1):9-18. doi:10.1513/pats.201201-014ST

5. Vercoulen JH, Daudey L, Molema J, Vos PJ, Peters JB, Top M, et al. An Integral assessment framework of health status in chronic obstructive pulmonary disease (COPD). Int J Behav Med. 2008;15(4):263-79. doi:10.1080/10705500802365474

6. Wilson IB, Cleary PD. Linking clinical variables with health-related quality of life. A conceptual model of patient outcomes. JAMA. 1995;273(1):59-65. doi:10.1001/jama.1995.03520250075037

7. Long Alliantie Nederland. Zorgstandaard astma volwassenen [Dutch guideline for adults with asthma]. [cited 2020 jun 22]. Available from: http://www.longalliantie.nl/files/4113/7335/4473/Zorgstandaard_Astma_Volwassenen.pdf. 
8. Long Alliantie Nederland. Zorgstandaard COPD [COPD guideline]. [cited 2020 jun 22]. Available from: http://www.longalliantie.nl/files/9014/5578/9160/LAN_Zorgstandaard_COPD-2016-2.pdf.

9. Long Alliantie Nederland. Leren van de koplopers: Implementatie van de zorgstandaarden astma en COPD, 2016 [To learn from the trendsetters: implementation of guidelines asthma and COPD, 2016]. [cited 2020 jun 22] Available from: http://www.longalliantie.nl/files/2614/6493/9103/Leren_van_de_Koplopers_-_enkel.pdf

10. van den Akker EF, van 't Hul AJ, Chavannes NH, Braunstahl GJ, van Bruggen A, Rutten-van Molken MP, et al. Development of an integral assessment approach of health status in patients with obstructive airway diseases: the CORONA study. Int J Chron Obstruct Pulmon Dis. 2015;10:2413-22. doi:10.2147/copd.S90680

11. van den Akker EF, Van't Hul AJ, Birnie E, Chavannes NH, Rutten-van Molken MP, In't Veen JC. Comprehensive Diagnostic Assessment of Health Status of Patients with Asthma or COPD: A Delphi Panel Study among Dutch Experts. Copd. 2017;14(2):190-9. doi:10.1080/15412555.2016.1264378

12. Agusti A, Bel E, Thomas M, Vogelmeier C, Brusselle G, Holgate $S$, et al. Treatable traits: toward precision medicine of chronic airway diseases. Eur Respir J. 2016;47(2):410-9. doi:10.1183/13993003.01359-2015

13. Long Alliantie Nederland. Multidisciplinaire richtlijn astma, 2014 [Multidisciplinary guideline asthma, 2014]. [cited 2020 jun 22 ]. Available from: http://www.longalliantie.nl/files/2313/9506/6963/Multidisciplinaire_richtlijn_astma_STATUS_GEAUTORISEERD_28012014_def.pdf.

14. vercoulen $\mathrm{JH}$. A simple method to enable patient-tailored treatment and to motivate the patient to change behaviour. Chron Respir Dis. 2012;9(4):259-68. doi:23129804

15. Herland K, Akselsen JP, Skjonsberg OH, Bjermer L. How representative are clinical study patients with asthma or COPD for a larger "real life" population of patients with obstructive lung disease? Respir Med. 2005;99(1):11-9. doi:10.1016/j.rmed.2004.03.026

16. McHugh. Interrater reliability: the kappa statistic. Biochem Med (Zagreb). 2012;22(3):276-82.

17. McDonald VM, Clark VL, Cordova-Rivera L, Wark PAB, Baines KJ, Gibson PG. Targeting Treatable Traits in Severe Asthma: A Randomised Controlled Trial. Eur Respir J. 2019. doi:10.1183/13993003.01509-2019

18. AM MPPBW. Motivation, Capacity and Opportunity to Complain: Towards a Comprehensive Model of Consumer Complaint Behavior. Advances in Consumer Research. 1997;24(1):464-9. doi:10.1108/09604520710834966

19. Hoff J. Lessen uit het mislukken van de parkinsonregistratie, 2019 [Lessons from the failed parkinsons registration, 2019]. [cited 2020 jun 22]. Available from: https://www.medischcontact.nl/nieuws/laatste-nieuws/artikel/lessen-uit-het-mislukken-van-deparkinsonregistratie.htm.

20. Rothnie KJ, Chandan JS, Goss HG, Mullerova H, Quint JK. Validity and interpretation of spirometric recordings to diagnose COPD in UK primary care. Int J Chron Obstruct Pulmon Dis. 2017;12:1663-8. doi:10.2147/copd.S133891

21. Schermer TR, Jacobs JE, Chavannes NH, Hartman J, Folgering HT, Bottema BJ, et al. Validity of spirometric testing in a general practice population of patients with chronic obstructive pulmonary disease (COPD). Thorax. 2003;58(10):861-6. doi:10.1136/thorax.58.10.861

\section{Tables}

Table 1; Data in table represent median (interquartile range) percentage of diagnostic items per patient.

\begin{tabular}{|lllll|}
\hline Domain & Baseline 2013 $(\mathrm{n}=101)$ & Care Pathway (2015) & Usual Care (2015) & Care pathway vs Baseline \\
\hline Physiological impairments & $0.61(.50-.67)$ & $0.78(.69-.83)$ & $0.72(.68-.78)$ & $\mathrm{p}<.001$ \\
\hline Symptoms & $0.75(.50-.75)$ & $1.0(.75-1.0)$ & $0.75(.75-.75)$ & $\mathrm{p}<.001$ \\
\hline Quality of life & $0(0-0)$ & $1.0(1.0-1.0)$ & $0.50(0.50-1.0)$ & $\mathrm{p}<.001$ \\
\hline Functional limitations & $0(0-0)$ & $0.67(67-.83)$ & $0.33(0-.33)$ & $\mathrm{p}<.001$ \\
\hline Overall & $0.52(.44-.59)$ & $0.81(.74-.85)$ & $0.69(.59-.74)$ & $\mathrm{p}<.001$ \\
\hline
\end{tabular}

Table 2; Data represent mean (SD) \% of filled-out diagnostic items per patient 


\begin{tabular}{|llll|}
\hline Domain & Care pathway (2015) & Usual Care in reference hospitals (2016) & Care Pathway vs usual care \\
\hline Physiological impairments & $0.77(.09)$ & 0.62 & $\mathrm{p}<.001$ \\
\hline Symptoms & $0.90(.14)$ & 0.56 & $\mathrm{p}<.001$ \\
\hline Quality of life & $0.98(.09)$ & 0.02 & $\mathrm{p}<.001$ \\
\hline Functional limitations & $0.75(.15)$ & 0.08 & $\mathrm{p}<.001$ \\
\hline Overall & $0.80(.07)$ & 0.51 & $\mathrm{p}<.001$ \\
\hline
\end{tabular}

\section{Figures}

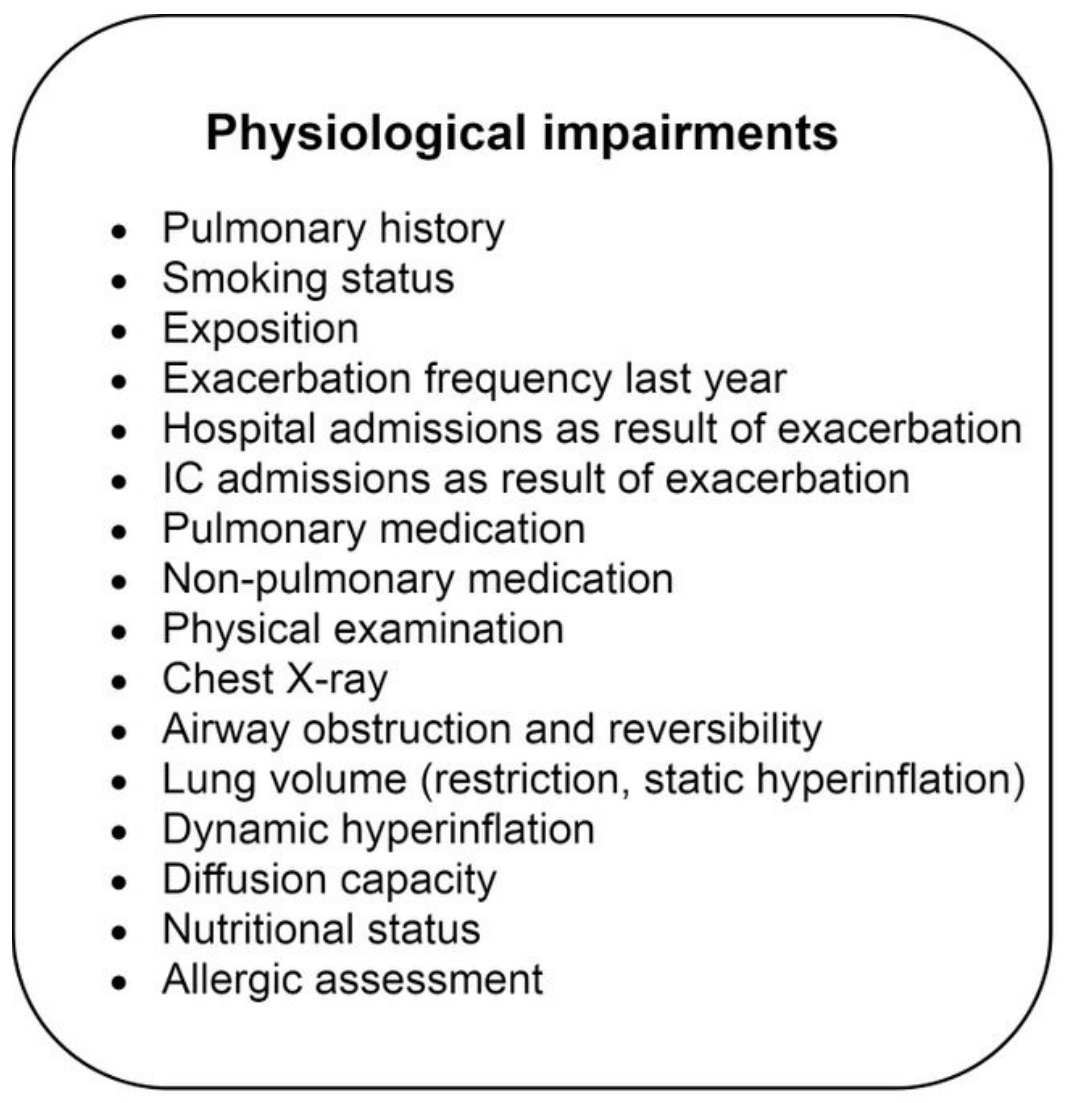

\section{Symptoms}

- Respiratory complaints

- Cardiac complaints

- Allergy subjective

- Anxiety and depression

\section{Functional limitations}

- Physical activity subjective

- Physical activity objective

- Exercise capacity

\section{Quality of life}

- Quality of life asthma

- Quality of life COPD

Figure 1

Diagnostic items per domain. 


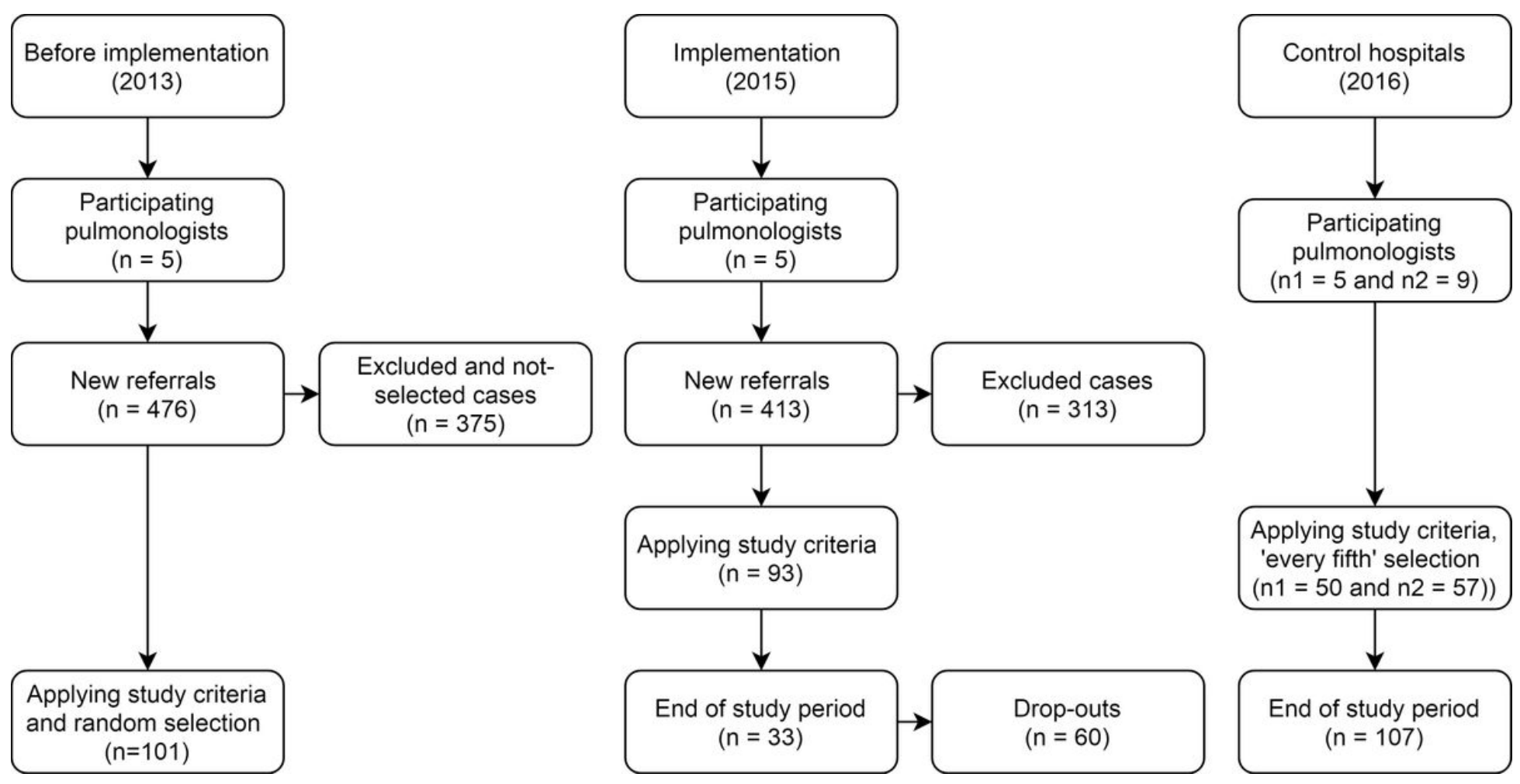

\section{Figure 2}

Flowchart of data entries.

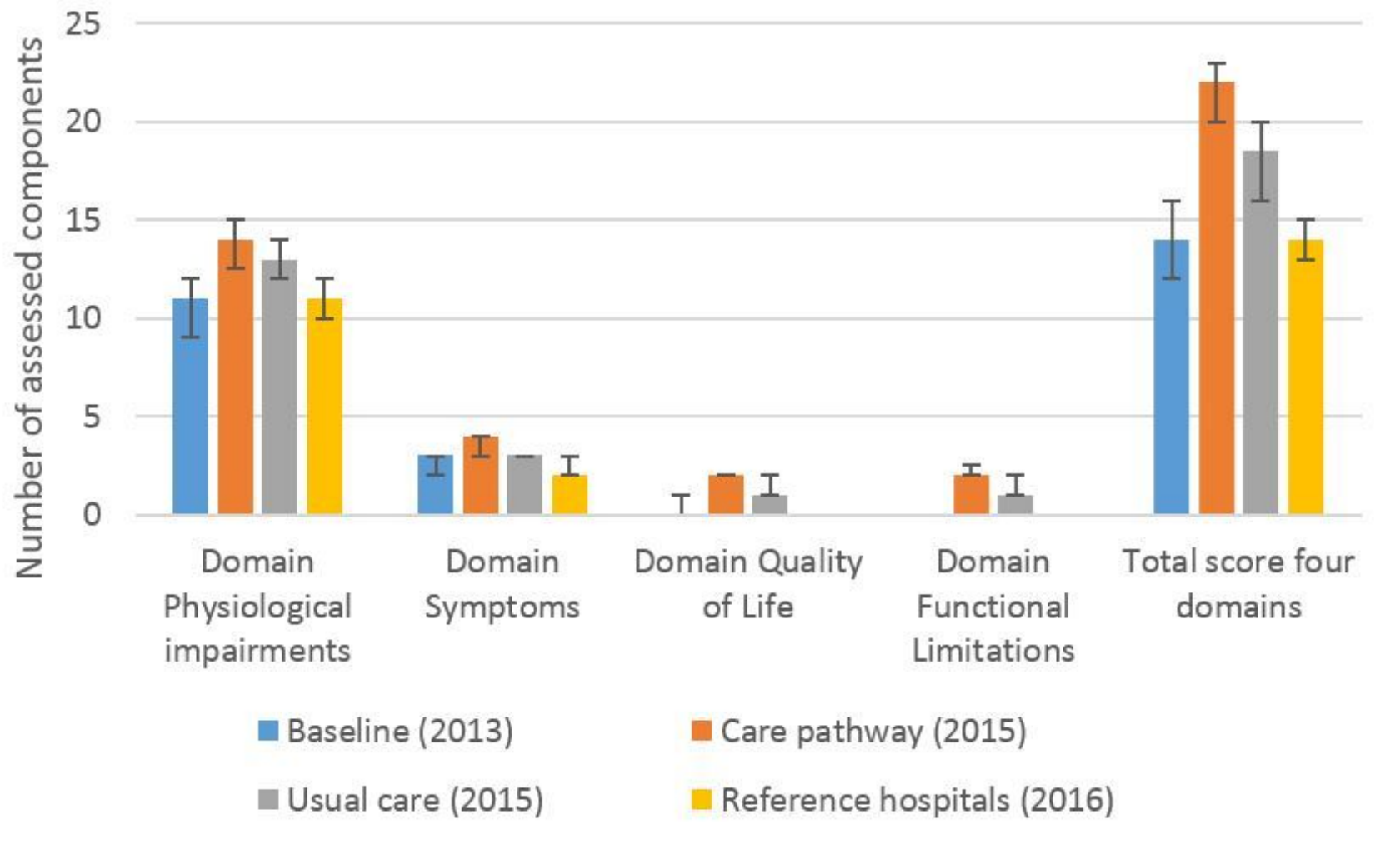

\section{Figure 3}

Mean number of components measured per domain. 


\section{Supplementary Files}

This is a list of supplementary files associated with this preprint. Click to download.

- STROBEchecklistv4combined.docx 